\title{
The behavior of solutions of nonlinear degenerate parabolic equations in nonregular domains and removability of singularity on boundary
}

\author{
Tahir S. Gadjiev * • Konul Mamedova
}

Received: 02.11.2019 / Revised: 28.07.2020 / Accepted: 11.09.2020

\begin{abstract}
In this paper the behavior of solutions of the initial-boundary problem of nonlinear degenerated parabolic equations of higer order in irregular domains and removability of singularity on boundary are studied. Analogies of those known as Saint-Venant's principle in theory of elasticity are obtained.
\end{abstract}

Keywords. Nonlinear parabolic equations, degenerate, Saint-Venant's principle, removability of singularity.

Mathematics Subject Classification (2010): 35J25, 35B40

\section{Introduction}

The Saint-Venant's principle is well-known in theory of elasticity. In many papers analogies of Saint-Venant's principle is used for investigation of the behavior of solutions partial differential equations. The analogies of Saint-Venant's principle are some of energy estimations for partial differential equations.

This method firstly is used in [1,2] and energy estimations for generalized solutions of second order linear equations were studied. We mention also papers [3-27].

Later in $[3,4,14]$ quality properties of solutions nonlinear equations are investigated - a method was devoloped for obtaining "increasing lemma" depending on the geometry of a domain. Otherwise these "increasing lemma" are energy apriori estimates of type of SaintVenant's principle.

In the study of quality properties of solutions of problems the role of the Saint-Venant's estimates is close to role of "increasing lemmas" of E.M. Landis [14] in analysis of properties continuously solutions of second order equations.

These estimates of type of Saint-Venant's principle allow us to study the behavior of solution in bounded domains with nonsmooth boundary, the behavior of solution in unbounded domains. The last gives of type of the Phragmén-Lindelöf theorems for behavior of solution in unbounded domains with noncompact boundary are obtained.

\footnotetext{
^ Corresponding author

T.S. Gadjiev

Institute of Mathematics and Mechanics of NAS of Azerbaijan, Az-1141, V. Bahabzade 9, Baku, Azerbaijan

E-mail: tgadiev@mail.az

K. Mamedova

Nakhchivan State University, Nakhchivan, Azerbaijan

E-mail: tgadiev@mail.az
} 
The Saint-Venant's estimates also allow us to get uniqueness theorems of generalized solutions.

With help of the Saint-Venant's estimates we can also get some theorems about removability singularity on baundary.

Therefore, it is very important to obtain Saint-Venant's estimates based on the geometry of domains.

We investigate equations in bounded domains with nonsmooth boundary, in unbounded domains with noncompact boundaries, also removable singularity at boundary.

\section{The behavior of solutions in domains with nonsmooth boundary}

Let us consider bounded domain $Q_{T}=\Omega \times(0, T), \Omega \subset \mathbb{R}^{n}, n \geq 2,0<T<\infty$, $\partial Q_{T}=\Gamma_{0} \cup \Gamma_{T} \cup \Gamma, \Gamma_{0}=\Omega \times\{0\}, \Gamma_{T}=\Omega \times\{T\}, \Gamma=\partial \Omega \times(0, T), \Omega$ - has a nonsmooth boundary. Nonsmoothness conditions will be given using a nonlinear frequency. Using a nonlinear frequency non-smooth domains are divided into two classes: narrow and wide domains.

The space $L_{p}\left(0, T, W_{q, \omega}^{m}\left(\Omega_{t}\right)\right)$ is determined as $\left\{u(x, t): \int_{0}^{T}\left(\|u\|_{W_{q, \omega}^{m}\left(\Omega_{t}\right)}\right)^{p} d t<\infty\right\}$, where $\Omega_{t}=Q_{T} \cap\{(x, \tau): \tau=t\}$ and $W_{q . \omega}^{m}\left(\Omega_{t}\right)$ - weighted Sobolev space such that weight $\omega$ belongs to the Muckenhoupt class (see [8]). The generalized solution is determined from the space $L_{p}\left(0, T, W_{q, \omega}^{m}\left(\Omega_{t}\right)\right) \cap W_{2}^{1}\left(0, T ; L_{2}(\Omega)\right)$.

We consider initial-boundary problem

$$
\begin{gathered}
\frac{\partial u}{\partial t}-\sum_{|\alpha| \leq 2 m}(-1)^{|\alpha|} D^{\alpha} A_{\alpha}\left(x, t, u, D u, \ldots, D_{x}^{m} u\right)=0, \\
\left.u\right|_{t=0}=0 \\
\left.D_{x}^{\alpha} u\right|_{\Gamma}=0,|\alpha| \leq m-1,
\end{gathered}
$$

where $D_{x}^{\alpha}=\frac{\partial^{|\alpha|}}{\partial x_{1}^{\alpha} \ldots \partial x_{n}^{\alpha}},|\alpha|=\alpha_{1}+\alpha_{2}+\ldots+\alpha_{n}, n \geq 1$.

Assume that the coefficients $A_{\alpha}(x, t, \xi)$ are measurable with respect to $(x, t) \in Q_{T}$, continuously with respect to $\xi \in \mathbb{R}^{M}$, where $M$ is the number of different multi-indices of length no more than $m$ and satisfying the conditions

$$
\begin{gathered}
\sum_{|\alpha| \leq m} A_{\alpha}(x, t, \xi) \xi_{\alpha}^{m} \geq C \omega(x)\left|\xi^{m}\right|^{p}-C_{1} \omega(x) \sum_{i=1}^{m-1}\left|\xi_{i}\right|^{p}-f_{1}(x, t) \\
\left|A_{\alpha}(x, t, \xi)\right| \leq C_{2} \omega(x) \sum_{i=0}^{m}\left|\xi_{i}\right|^{p}+f_{2}(x, t),
\end{gathered}
$$

where $\xi=\left(\xi^{0}, \ldots, \xi^{m}\right), \xi^{i}=\left(\xi_{\alpha}^{i}\right),|\alpha|=i, \quad p>1$,

$$
\begin{gathered}
f_{1} \in L_{p}\left(0, T, L_{p, l o c}\left(\Omega_{t}\right)\right), \\
f_{2} \in L_{1, l o c}\left(\Omega_{T}\right) .
\end{gathered}
$$
norm

The space $W_{q, \omega}^{m}\left(\Omega_{t}\right)$ is the closure of $\Omega_{t}$ the functions from $C^{m}(\bar{\Omega})$ with respect to the

$$
\|u\|_{W_{q, \omega}^{m}\left(\Omega_{t}\right)}=\left(\int_{\Omega_{t}} \omega(x) \sum_{|\alpha| \leq m}\left|D_{x}^{\alpha} u(x, t)\right|^{q} d x d t\right)^{1 / q} .
$$


Now we describe the geometry of $Q_{T}$ with nonlinear frequency $\lambda_{p}^{p}(r, \tau)$ of section $\sigma(r, \tau)=S(r) \cap \Omega_{\tau}$, where $S(r)=Q_{T} \cap \partial Q_{T}(r), Q_{T}(r)=Q_{T} \cap B_{r} \times(0, T), B_{r}=$ $B(0, r)$ is the ball with radius $r$ centered at 0 . Thus

$$
\lambda_{p}^{p}(r, \tau)=\inf \left(\int_{\sigma(r, \tau)} \omega(x)\left|\nabla_{s} v\right|^{p} d \tau\right)\left(\int_{\sigma(r, \tau)} \omega(x)|v|^{p} d \tau\right)^{-1},
$$

where the lower bound is taken by all continuously differentiable functions in the vicinity of $\sigma(r, \tau)$ that vanish on $\partial Q_{T} ; \nabla_{s} v(x)$ is a projection of the vector $\nabla v(x)$ on a tangential plane to $\sigma(r, \tau)$ at the point $x$. The function $u \in L_{p}\left(0, T ; \stackrel{\circ}{W^{m}}{ }_{p, \omega, l o c}\left(\Omega_{t}\right)\right) \cap W_{2}^{1}\left(0, T ; L_{2, l o c}\left(\Omega_{t}\right)\right)$ is said to be a generalized solution of the problem (2.1)-(2.2) if the integral identity

$$
\int_{Q_{T}} \frac{\partial u}{\partial t} \varphi d x d t+\int_{Q_{T}} \sum_{|\alpha| \leq m} A_{\alpha}\left(x, t, u, D u, \ldots, D^{m} u\right) D^{\alpha} \varphi d x d t=0
$$

is fulfilled for the arbitrary function $\varphi \in L_{p}\left(0, T ; \stackrel{\circ}{\stackrel{\circ}{W}^{m}} p, l_{\text {, }}\left(\Omega_{t}\right)\right) \cap L_{2}\left(Q_{T}\right)$.

Generally we will consider classes of domains, for which the following estimate

$$
\int_{S_{r}} \omega(x)|u(x, t)|^{p} d x d t \leq \lambda_{p}^{-p}(r) \int_{S_{r}} \omega(x)|\nabla u(x, t)|^{p} d x d t
$$

holds. The necessary and sufficiently conditions on domains for holds estimate (2.7) is given in [9]. Using a nonlinear frequency we divide non-smooth domains into two classes:

1. The first class is the narrow domain class, i.d. complement of neighborhood of a point is sufficiently massive. For example, this class contains some cone with a vertex at this point. In terms of nonlinear frequency this class of domains satisfies the condition

$$
r \lambda_{p}(r)>d_{1}>0, \forall r \in\left(0, r_{0}\right), r_{0}>0 .
$$

2. The second class is the broad domain class, i.d. those points, whose complement of neighborhood of a point is rather narrow. In terms of the nonlinear frequency, this class of regions satisfies the condition

$$
r \lambda_{p}(r)<d_{2}<\infty, \forall r \in\left(0, r_{0}\right) .
$$

Also the function $\varphi(r)$ is determined at $\left(0, r_{0}\right)$ by inequality

$$
\inf _{r \psi(r)<|x|<r} \lambda_{p}(|x|)(r-r \psi(r)) \omega(x) \geq \mu>0,
$$

where $0<1-c_{0}<\mu<1$.

Let

$$
\begin{gathered}
J(r)=\int_{\Omega_{r}} \omega(x)\left|D^{m} u(x, t)\right|^{p} d x d t \\
G(r)=\int_{\Omega_{r}}\left(\sum_{|\alpha| \leq m} \omega(x)\left(\left|F_{\alpha}(x, t)\right|+\left|f_{2}(x, t)\right|\right)^{\frac{p}{q}} \lambda_{p}^{-\frac{m-|\alpha|}{p-1} p}(|x|)+\left|f_{1}(x, t)\right|\right) d x d t .
\end{gathered}
$$

As mentioned above, Saint-Venant type estimates are needed first to get the required estimates. Further using various lemmas we get estimates in bounded domains with non-smooth boundary, in unbounded domains with noncompact boundary and removable singularity 
on boundary in bounded domains. In unbounded domains we get theorems of PhragménLindelöf type.

Therefore, we give the following lemma for the case of bounded domains.

Let $0<\varphi_{1}(r)<c_{0}<1$ be a measurable function on interval $\left(0, r_{0}\right)$ and $r_{0}$ sufficiently small.

Lemma 2.1 Assume that $J(r)$ is a continuous nondecreasing function on $\left(0, r_{0}\right)$ and satisfies the inequality

$$
J\left(r\left(1-\varphi_{1}(r)\right)\right) \leq \lambda J(r)+h(r), \forall r \in\left(0, r_{0}\right),
$$

where $0<\lambda<1$ and $h(r)$ is bounded.

Then the estimation

$$
J(r) \leq C \exp \left(-\delta \ln \frac{1}{\lambda} \int_{r}^{r_{0}} \frac{d \tau}{\tau \varphi_{1}(\tau)}\right)\left(J\left(r_{0}\right)+h\left(r_{0}\right)\right)
$$

is valid for $J(r)$. Here $0<\delta<1-c_{0}$.

The proof of this lemma can be found in $[3,4,11]$.

We define a function $\psi(r)$ on $\left(0, r_{0}\right)$ by the inequlaity

$$
\inf _{r \psi(r)<|x|<r} \lambda_{p}(|x|)(r-r \psi(r)) \omega(x) \geq \mu>0,
$$

where $\mu$ is such that $0<1-c_{0}<\psi(r)<1$.

Theorem 2.1 Let $u \in L_{p}\left(0, T ; \stackrel{\circ}{W_{p, \omega, l o c}^{m}}\left(\Omega_{t}\right)\right) \cap W_{2}^{1}\left(0, T ; L_{2}\left(\Omega_{T}\right)\right)$ be the generalized solution of problem (2.1)-(2.3). Assume that coefficients satisfy the conditions (2.4) and (2.5), the domain satisfies the condition $(A)$, the weight satisfies the Muckenhoupt condition, the function $\psi(r)$ satisfies condition (2.10) and $G(r)$ is bounded.

Then for $J(r)$ the following estimate holds

$$
J\left(r \exp \left(-\frac{1-\psi(r)}{1-c_{0}-\theta}\right)\right) \leq C \exp \left(-\theta \ln \frac{1}{\nu} \int_{r}^{r_{0}} \frac{d \tau}{\tau 1-\psi(r)}\right)\left(J\left(r_{0}\right)+G\left(r_{0}\right)\right)
$$

for every $\nu>0$ and $\theta<1-c_{0}$.

Proof. This theorem is Saint -Venant's type estimate. For proof we substitute special test functions to integral identity (2.6) and do some calculations (see also [4]).

Corollary 2.1 This estimate is new in the case of linear equations of following type

$$
\begin{gathered}
\frac{\partial u}{\partial t}-\sum_{|\alpha| \leq m} a_{\alpha}(x, t) D^{\alpha} u=\sum_{|\alpha| \leq m} D^{\alpha} F_{\alpha}(x, t), \\
c_{1} \omega(x)|\xi|^{2 m} \leq \sum_{|\alpha|=m} a_{\alpha} \xi^{\alpha} \leq c_{2} \omega(x)|\xi|^{2 m}, \quad x \in \Omega, \xi \in \mathbb{R}^{n},
\end{gathered}
$$

$c_{1}, c_{2}>0, F_{\alpha} \in L_{2}\left(\Omega_{t}\right), a_{\alpha} \in C^{|\alpha|-m}\left(\overline{Q_{T}}\right)$ at $|\alpha|>m$ and $a_{\alpha}(x, t)$ is a measurable function such that $|\alpha| \leq m$. 
Corollary 2.2 Let $u \in L_{p}\left(0, T ; \stackrel{\circ}{W}_{p, \omega, l o c}^{m}\left(\Omega_{t}\right)\right) \cap W_{2}^{1}\left(0, T ; L_{2}\left(\Omega_{t}\right)\right)$ be generalized solution of problem (2.1)-(2.3) and $0 \in \partial \Omega$. The domain $\Omega$ in the neighborhood of the point has a boundary such that $\lambda_{p}(r)>\lambda^{(0)} r^{-1}$ for any $r \in\left(0, r_{0}\right), \lambda^{(0)}>0$. Then there exists $\gamma_{0}>0$ such that if for $G(r)$ the following estimate holds

$$
G(r) \leq C r^{\gamma_{0}+\varepsilon} G\left(r_{0}\right), \forall r \in\left(0, r_{0}\right)
$$

for sufficiently small $\varepsilon$, the following estimate holds

$$
\omega(x)\left|D^{j} u(x, t)\right| \leq C|x|^{m-\frac{n}{p}-j+\gamma_{0}}(J(r)+G(r))^{\frac{1}{p}},
$$

$j=0,1, \ldots,\left[m-\frac{n}{p}\right]$.

The proof of this corollary follows from estimate (2.11) and embedding theorems.

\section{The removable singularity of solutions}

First we state an auxiliary lemma.

Lemma 3.1 Let $I(r)$ be a non-negative and non-growing function on interval $\left(0, r_{0}\right), r_{0}>$ 0 , function satisfying condition

$$
I(r) \leq \theta I(r \varepsilon(r))+G(r \varepsilon(r)), 0<\theta<1,
$$

where $\varepsilon(r)$ is a measurable function, $0<c_{0}<\varepsilon(r)<1$ such that

$$
k(r)=(\varphi(r))^{-1} \inf _{r \varepsilon(r)<\tau<r} \varphi(\tau) \geq \nu>0, \varphi(r) \equiv 1-\varepsilon(r)
$$

and function $G(r)$ is a measurable and locally bounded.

Then the following holds:

1) $I\left(r_{i}\right)<c G\left(r_{i}\right)$ for some sequence $r_{i} \rightarrow 0$;

2) or I(r) is growing fast enough as $r \rightarrow 0$, namely

$$
I(r) \geq C \exp \left(\ln (\theta+\delta)^{-1} \int_{r}^{r_{0}} \frac{d \tau}{\tau(1-\varepsilon(r))}\right) I\left(r_{0}\right),
$$

where $0<\delta<1-\theta$.

See [10] for the proof of this lemma.

We will define

$$
I(r)=\int_{\Omega \backslash \Omega_{r}} \omega(x)\left|D^{m} u\right|^{p} d x d t .
$$

For small $r$, for the behavior $I(r)$ following theorem holds. This theorem gives apriori estimates of energy integrals.

Theorem 3.1 Let $u \in L_{p}\left(0, T ; \stackrel{\circ}{W_{p, \omega}^{m}}\right.$ loc $\left.(\Omega, \Gamma)\right) \cap W_{2}^{1}\left(0, T ; L_{2}\left(\Omega_{T}\right)\right)$ be generalized solution of problem (2.1)-(2.3). Assume that coefficients satisfy the conditions (2.4) and (2.5), the domain satisfies the condition $(A)$, the weight satisfies the Muckenhoupt condition, the function $\psi(r)$ satisfies condition (2.10) and $k(r)$ satisfies condition (3.2). Then for $I(r)$ the following holds: 
1) $I\left(r_{i}\right) \leq c\left(1+G\left(r_{i}\right)\right)$ for some sequence $r_{i} \rightarrow 0$;

2) or $I(r)$ is growing fast enough as $r \rightarrow 0$, namely

$$
I(r) \leq C \exp \left(\ln \frac{1}{k_{0}+\delta} \int_{r}^{r_{0}} \frac{d \tau}{\tau \psi(r)}\right),
$$

where $k_{0}=$ const.

Also the following theorem holds.

Theorem 3.2 Let $u \in L_{p}\left(0, T ; \stackrel{\circ}{W}_{p, \omega, l o c}^{m}(\Omega, \Gamma)\right) \cap W_{2}^{1}\left(0, T ; L_{2}\left(\Omega_{T}\right)\right)$ be generalized solution of problem (2.1)-(2.3). In the conditions of Theorem 3.1 the following inequality holds

$$
I(r) \leq C \exp \left(-c \int_{r}^{r_{0}} \frac{\psi(\tau) \tau^{-1}}{1+\psi(\tau)} d \tau\right), \forall r<r_{0} .
$$

Then singularity set $\Gamma$ of solution $u(x, t)$ removable, i.d. $u \in L_{p}\left(0, T ; \stackrel{\circ}{W}_{p, \omega}^{m}(\Omega)\right) \cap$ $W_{2}^{1}\left(0, T ; L_{2}(\Omega)\right)$.

The proof of Theorems 3.1 and 3.2 similarly to correspondingly theorems from $[4,10,11]$.

These theorems generalize the corresponding results in $[12,14,16-19]$.

\section{The behavior solutions in unbounded domains. Theorem of types Phragmén-Lindelöf}

Now we consider unbounded domains. First we define a measurable function $\psi(r): 1<$ $\psi(r)<\infty, \forall r>r_{0}>0$ and $\varphi_{0}$ is continuous, non-growing function such that $\varphi_{0}(r) \geq$ $r^{-1} \sup (h(r))-1$ at $r>r_{0}$. Here the upper bound is taken over all non-decreasing function $h(r): h(r) \leq r \psi(r)$ at $r_{0}<r<\infty$.

Lemma 4.1 Assume that on $\left(r_{0}, \infty\right)$ non-negative, continuous function $I(r)$ satisfies inequality

$$
I(r) \leq \theta I(r \psi(r)), \psi(r)=1+\varphi_{0}(r)
$$

for all $r \in\left(r_{0}, \infty\right)$ and $0<\theta<1$. Then for any $r \in\left(r_{0}, \infty\right)$ the following estimate holds

$$
I\left(r \exp \left(-\frac{\varphi_{0}(r)}{1-\nu}\right)\right) \geq \theta \exp \left(\nu \ln \theta^{-1} \int_{r_{0}}^{r} \frac{d \tau}{\tau \varphi_{0}(\tau)}\right) I\left(r_{0}\right)
$$

for all $\nu \in(0,1)$.

The proof of Lemma 4.1 is similar to the proof of the corresponding result in [11].

The unbounded domains which satisfy isoperimetric conditions divided into two classes, depending on behavior nonlinear frequency function $\lambda_{p}(r)$ at $r \rightarrow \infty$. First class is narrow domains and in our terms this condition is

$$
r \lambda_{p}(r)>c>0, \forall r>r_{0}>0 .
$$

Second class is wide domains and in our terms this condition is

$$
r \lambda_{p}(r) \leq c<\infty, \forall r>r_{0}>0 .
$$


Also we define the function $\psi(r)$ for $h_{0}>0$ by inequality

$$
\inf _{r<\tau<r \psi(r)} r \lambda_{p}(\tau)(\psi(r)-1) \geq h_{0}, \psi(r)>1, \forall r>r_{0} .
$$

Let

$$
\begin{gathered}
J(r)=\int_{\Omega_{r}} \omega(x)\left|D^{m} u(x, t)\right|^{p} d x d t \\
G(r)=\int_{\Omega_{r}}\left(\sum_{|\alpha| \leq m}\left(\left|F_{\alpha}(x, t)\right|+\left|f_{2}(x, t)\right|\right)^{\frac{p}{p-1}} \lambda_{p}^{-\frac{m-|\alpha|}{p-1} p}(|x|)+\left|f_{1}(x, t)\right|\right) d x d t .
\end{gathered}
$$

The following theorem is true.

Theorem 4.1 Let $u \in L_{p}\left(0, T ; \stackrel{\circ}{W_{p, \omega}^{m}(x), l o c}\left(\Omega_{t}\right)\right) \cap W_{2}^{1}\left(0, T ; L_{2}\left(\Omega_{T}\right)\right)$ be generalized solution of problem (2.1)-(2.3), $\omega(x)$ be in Muckenhoupt classes. Assume that coefficients satisfy the conditions (2.4) and (2.5). Moreover domain $\Omega$ is narrow enough in the sense that $\lambda_{p}(r)>\delta^{-1}$ at $\left(r_{0}, \infty\right)$. Let $\psi(r)$ be any function which satisfy condition (4.3) and $\varphi_{0}(r)$ be the function under construction by $\psi(r)$ as in Lemma 4.1. Then for $J(r)$ the following hold:

1.

$$
\lim _{r \rightarrow \infty} \frac{J(r)}{G(r)}<\infty
$$

2. $\mathrm{Or}$

$$
J\left(r \exp \left(\frac{\varphi_{0}(r)}{1-\nu}\right)\right) \geq \theta \exp \left(\nu \ln \frac{1}{\theta} \int_{r_{0}}^{r} \frac{d \tau}{\tau \varphi_{0}(\tau)}\right) J\left(r_{0}\right)
$$

for all $\nu \in(0,1)$ and for all $r>r_{0}$ at big enough $r_{0}$.

The proof of Theorem 4.1 is similar the proof of the corresponding result in [11].

Corollary 4.1 This is Phragmén-Lindelöf type theorem. Also we can give this type theorems for wide classes of domains and for integrals of functions.

Corollary 4.2 Many examples can be given showing the sharpness of the results obtained in different domains.

\section{Acknowledgements}

The authors thank the referee(s) for careful reading the paper and useful comments.

\section{References}

1. Oleinik, O.A., Iosif'yan, G.A.: Boundary value problems for second order elliptic equations in unbounded domains and Saint-Venant's principle, Ann. Scuola Norm. Sup. Pisa Cl. Sci. (4) 4 (2), 269-290 (1977)

2. Oleinik, O.A., Iosif'yan, G.A.: Removable boundary singularities and uniqueness of solutions of boundary value problems for second order elliptic and parabolic equations, (Russian) Funkcional. Anal. i Priloen. 11 (3), 54-67 (1977)

3. Tedeev, A.F., Shishkov, A.E.: Qualitative properties of solutions and subsolutions of quasilinear elliptic equations, Izv. Vyssh. Uchebn. Zaved. Mat. (Russian) 1, 62-68 (1984) 
4. Gadjiev, T.S.: On the behavior of solutions of a mixed boundary value problem for higher-order quasilinear elliptic equations of divergence type, (Russian) Differentsial'nye Uravneniya 26 (4), 703706 (1990)

5. Bonafade, S.: Quazilinear degenerate elliptic variational inequalities with discontinuous coefficients, Comment. Math. Univ. Carolinae 34 (1), 55-61 (1993)

6. Lancaster, K., Stanley, J.: On the asymptotic behavior of solutions of quasilinear elliptic equations, Ann. Univ. Ferrara-Sez. VII-Sc. Mat., vol. IL, 85-125 (2003)

7. Jorge, Garcia-Melian: Boundary behavior for large solutions to elliptic equations with singular weights, Nonlinear Analysis: Theory, Methods and Applications 67 (3), 818826 (2007)

8. Chanillo, S., Wheeden, R.: Weighted Poincare and Sobolev inequalities and estimates for weighted Peano maximal functions, Mer. J. Math. 107, 1191-1226 (1985)

9. Miklyukov, V.M.: On asymptotic properties of subsolutions of elliptic type quasilinear equations, (Russian) Mat. Sbor. 111(145), No 1, 42-66 (1980)

10. Gadjiev, T.S., Sadigova, N.R., Rasulov, R.A.: Removable singularities of solutions of degenerate nonlinear elliptic equations on the boundary of a domain, Nonlinear Anal. 74 (16), 5566-5571 (2011)

11. Gadjiev, T.S., Mamedova, K.N.: On behavior of solutions degenerate parabolic equations, Trans. Natl. Acad. Sci. Azerb. Ser. Phys.-Tech. Math. Sci. 32 (4), Mathematics and Mechanics, 43-50 (2012)

12. Carleson, L.: Selected Problems on Exceptional Sets, D. Van Nostrand Company, Toronto, London, Melbourne (1967)

13. Kondratyev, V.A., Oleynik, O.A.: On the behavior of generalized solutions of the Dirichlet problem for high-order elliptic equations in the neighborhood of a boundary, (Russian) Boundary value problems of mathematical physics and related questions in the theory of functions, 14. Zap. Nauchn. Sem. Leningrad. Otdel. Mat. Inst. Steklov. (LOMI) 115, 114-125 (1982)

14. Kondratyev, V.A., Landis, E.M.: Qualitative theory of partial differential equations of second order, (Russian) (Russian) Partial differential equations, 3 (Russian), 99215, 220, Itogi Nauki i Tekhniki, Sovrem. Probl. Mat. Fund. Naprav., 32, Akad. Nauk SSSR, Vsesoyuz. Inst. Nauchn. i Tekhn. Inform., Moscow (1988)

15. Gilbarg, D., Trudinger, N.: Elliptic Partial Differential Equations of Second Order, Springer-Verlag, Berlin, New York (1977)

16. Gadjiyev, T.S., Mamedova, V.: On removable sets of solutions of the second order elliptic and parabolic equations in nondivergent form, Ukrainian Math. J. 61 (11), 1743-1756 (2009)

17. Diederich, J.: Removable singularities of solutions of elliptic partial differential equations, Trans. Amer. Math. Soc. 165, 333-352 (1972)

18. Harvey, R., Polking, J.: Removable singularities of solutions of linear partial differential equations, Acta Math. 125, 39-56 (1970)

19. Kilpelainen, T., Zhong, X.: Removable sets for continuous solutions of quasilinear elliptic equations, Proc. Amer. Math. Soc. 130 (6), 1681-1688 (2002)

20. Chanillo, S., Wheeden, R.: Weighted Poincare and Sobolev inequalities and estimates for weighted Peano maximal functions, Amer. J. Math. 107, 1191-1226 (1985)

21. Miklyukov, V.M.: Asymptotic properties of subsolutions of quasilinear equations of elliptic type and mappings with bounded distortion, (Russian) Mat. Sb. (N.S.) 111 (153) (1), 4266 (1980)

22. Gadjiyev, T.S., Mamedova, K.N.: On behavior of solutions degenerate parabolic equations, Trans. Natl. Acad. Sci. Azerb. Ser. Phys.-Tech. Math. Sci. 32 (4), Mathematics and Mechanics, 43-50 (2012)

23. Alikakos, N.D., Rostamian, R.: Gradient estimates for degenerate diffusion equations, Math. Ann. 259 (1), 5370 (1982) 
24. Guliyev, V.S., Gadjiev, T.S., Galandarova, Sh.: Dirichlet boundary value problems for uniformly elliptic equations in modified local generalized Sobolev-Morrey spaces, Electron. J. Qual. Theory Differ. Equ. Paper No. 71, 17 pp. (2017)

25. Gadjiev, T.S., Galandarova, Sh., Guliyev, V.S.: Regularity in generalized Morrey spaces of solutions to higher order nondivergence elliptic equations with VMO coefficients, Electron. J. Qual. Theory Differ. Equ. Paper No. 55, 17 pp. (2019)

26. Gadjiev, T.S., Guliyev, V.S., Suleymanova, K.G.: The Dirichlet problem for the uniformly elliptic equation in generalized weighted Morrey spaces, Studia Sci. Math. Hungar. 57 (1), 68-90 (2020)

27. Guliyev, V.S., Gadjiev, T.S., Serbetci, A.: The Dirichlet problem for the uniformly higher-order elliptic equations in generalized weighted Sobolev-Morrey spaces, Nonlinear Stud. 26 (4), 831-842 (2019) 